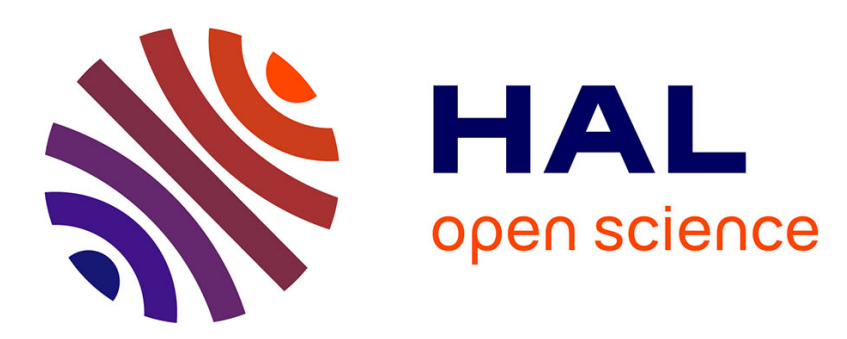

\title{
Les oïdiums des Cucurbitacées. I. - Données bibliographiques. Travaux préliminaires
}

Paul-Michel Molot, Hervé Lecoq

\section{To cite this version:}

Paul-Michel Molot, Hervé Lecoq. Les oïdiums des Cucurbitacées. I. - Données bibliographiques. Travaux préliminaires. Agronomie, 1986, 6 (4), pp.355-362. 10.1051/agro:19860405 . hal-02727800

\section{HAL Id: hal-02727800 \\ https://hal.inrae.fr/hal-02727800}

Submitted on 2 Jun 2020

HAL is a multi-disciplinary open access archive for the deposit and dissemination of scientific research documents, whether they are published or not. The documents may come from teaching and research institutions in France or abroad, or from public or private research centers.
L'archive ouverte pluridisciplinaire HAL, est destinée au dépôt et à la diffusion de documents scientifiques de niveau recherche, publiés ou non, émanant des établissements d'enseignement et de recherche français ou étrangers, des laboratoires publics ou privés. 


\section{Les oïdiums des Cucurbitacées. I. - Données bi- bliographiques. Travaux préliminaires}

Paul Michel MOLOT \& Hervé LECOQ

I.N.R.A., Station de Pathologie végétale, Centre de Recherches d'Avignon, F 84140 Montfavet

\begin{abstract}
Sur Cucurbitacées (concombre, courgette, melon, pastèque), il existe 3 oïdiums : Sphaerotheca fuliginea, Erysiphe cichoracearum, Leveillula taurica.

En fait, seuls les 2 premiers sont importants dans les conditions de la pratique agricole en France.

Différents critères d'identification concernant la forme conidienne sont passés en revue : forme des spores, disposition du tube germinatif, présence de grains de fibrosine, aspect de l'appressorium, etc...

Les conditions sèches sont plus favorables au développement et à la sporulation d'E. cichoracearum qu'à ceux de $S$. fuliginea qui semble davantage inféodé aux cultures sous abri.

Sous serre, $S$. fuliginea se développe mieux sur les plantes recevant une importante alimentation minérale. En revanche, une préinoculation virale (CMV, WMV 1, WMV 2 et ZYMV) confère au végétal un niveau de sensibilité plus faible.

En dépit d'une spécificité assez étroite, il existe des possibilités de contaminations croisées entre différentes plantes.

Le problème principal pour l'étude de ces champignons demeure celui de la conservation des souches pour la production d'un inoculum standard.
\end{abstract}

Mots clés additionnels : Sphaerotheca fuliginea, Erysiphe cichoracearum, Leveillula taurica, facteurs climatiques, alimentation de la plante, préinoculation virale, contaminations croisées, conservation des souches.

\begin{abstract}
Powdery mildews of cucurbits. I. Bibliographical review and preliminary experimental results.
Three different species of powdery mildews are commonly described on cultivated cucurbits : Sphaerotheca fuliginea, Erysiphe cichoracearum and Leveillula taurica. Only the first two are of major economic importance in France. The different ways of identifying these species according to their conidia are reviewed : spore shape, position of the germ tube, presence of fibrosine bodies, appressorium shape. Dry conditions seem to be more favourable for the growth and sporulation of E. cichoracearum, while $S$. fuliginea requires more humidity and is therefore very frequent in protected crops. Under greenhouse conditions good plant nutrition seems to promote development of $S$. fuliginea. In contrast, infection by different viruses seems to decrease susceptibility to the fungus. In spite of some host specificity, cross contamination between different hosts can be observed. The lack of an easy method for preserving powdery mildew strains remains a major difficulty for the study of the diseases concerned.
\end{abstract}

Additional key words : Sphaerotheca fuliginea, Erysiphe cichoracearum, Leveillula taurica, climatic conditions, plant nutrition, effect of virus infection, cross contamination, strain preservation.

\section{INTRODUCTION}

Dans la pratique agronomique, les oïdiums des Cucurbitacées revêtent, depuis quelques années, une importance sans cesse grandissante.

Cette situation, assez alarmante, résulte vraisemblablement de l'intervention de plusieurs facteurs : intensification des techniques culturales, absence de variétés commerciales résistantes, confusion fréquente des principaux genres et espèces d'oïdiums dont les biologies différentes exigent des techniques d'intervention adaptées, etc... Il est à noter que l'abus des traite- ments fongicides a peut-être conduit à l'apparition de phénomènes de résistance et à la multiplication de races physiologiques.

En culture protégée, là où existent des possibilités de lutte biologique à l'aide de Phytoseiulus persimilis Athias-Henriot (Acari : Phytoseiidae) contre les acariens et d'Encarsia formosa Gahan (Hymenoptera : Aphelinidae) contre les aleurodes des serres, la situation est encore aggravée par le fait que les produits anti-oïdiums, appliqués de façon intensive, contrarient l'efficacité du dispositif de lutte intégrée.

Plusieurs oïdiums sont classiquement reconnus sur 
Cucurbitacées (BAllantyne, 1975; SitTERly, 1978) :

Sphaerotheca fuliginea (Schlecht. \& Fr.) Poll. Erysiphe cichoracearum D.C. \& Mérat

Leveillula taurica (Lev.) Arnaud

mais seuls $S$. fuliginea et $E$. cichoracearum sont très fréquents (HIRATA, 1968 ; NAGY, 1970 ; CrUGER \& MEYER, 1976 ; ANGELOV, 1979 ; KHAN, 1983).

Ces différents oïdiums peuvent s'observer sur de nombreuses plantes sauvages ou cultivées (VIENNOTBOURGIN, 1956) mais tous possèdent une haute spécificité, les formes inféodées à un hôte donné n'infectant pas, en général, une autre espèce. C'est ainsi que $S$. fuliginea se rencontre sur Légumineuses (haricot), sur ricin et sur de nombreuses plantes spontanées (VIENNOT-BOURGIN, 1949, 1956), E. cichoracearum sur Solanacées (tabac, pomme de terre, tomate) et sur Composées (chicorée, laitue, pissenlit, topinambour, scorsonère, aster, souci, artichaut, etc...), L. taurica sur Solanacées (piment, tomate), sur Composées (artichaut) et sur de nombreuses autres familles.

D'après les observations effectuées dans divers pays, notamment en Hongrie (NAGY, 1970), en France (VIENNOT-BOURGIN, 1971), en Allemagne de l'Est (JANKE et al., 1977), en Bulgarie (ANGELOV, 1979 ; ANGelov \& Pethova, 1979) et en Tchécoslovaquie (LEBEDA, 1983), S. fuliginea colonise les Cucurbitacées aussi bien en plein champ qu'en serre. En revanche, E. cichoracearum s'observe surtout en cultures de plein air (SCHLÖSSER, 1976).

L. taurica a surtout été signalé sur concombre (TARR, 1955 ; GOLOVIN, 1956 ; GORDEEVA, 1961 ; TRAMIER, 1963 ; HIRATA, 1968 ; PYZHENKOV, 1977 ; COLLINGWOOD, 1977 ; El AMMARI \& KHAN, 1983 ; KHAN, 1983). En Roumanie, il est fait mention de L. taurica f. sp. cucumis sativi (DOCEA \& FRATILA, 1980).

Deux autres oïdiums, E. polygoni (DC.) St.-Am. et $E$. polyphaga Hammarlund, ont été décrits chez les Cucurbitacées. Le $1^{\text {er }}$, classiquement inféodé aux Légumineuses (pois, vesce, gesse, soja, luzerne, trèfle, lupin), mais mentionné sur divers Cucumis et Cucurbita en Pologne (SCHROETER, 1893) et au Japon (HOMMA, 1937), est actuellement contesté sur Cucurbitacées en raison de confusion taxonomique (KHAN, 1983). Le $2^{\text {e }}$ décrit pour la première fois par HAMMARLUND (1945) ne semble pas correspondre à une espèce séparée : tous les Erysiphe classés dans l'espèce polyphaga appartiennent en fait à l'espèce cichoracearum (NAGY, 1975).

\section{TABLEAU 1}

Mise en évidence par une gamme d'hôtes différentiels de melons de 3 races physiologiques chez Sphaerotheca fuliginea (modifié d'après THOMAS, 1978).

Physiological races of Sphaerotheca fuliginea according to the reaction of differential hosts (from THOMAS, 1978).

\begin{tabular}{lcccc}
\hline \hline \multicolumn{1}{c}{ Melons } & Race 1 & Race 2 & Race 3 & $\begin{array}{c}\text { Isolat de } \\
\text { Montfavet }\end{array}$ \\
\hline Védrantais & $\mathrm{S}$ & $\mathrm{S}$ & $\mathrm{S}$ & $\mathrm{S}$ \\
PMR 45 & $\mathrm{R}$ & $\mathrm{S}$ & $\mathrm{S}$ & $\mathrm{S}$ \\
PMR 5 & $\mathrm{R}$ & $\mathrm{R}$ & $\mathrm{S}$ & $\mathrm{R}$ \\
Edisto 47 & $\mathrm{R}$ & $\mathrm{S}$ & $\mathrm{R}$ & $\mathrm{R}$ \\
\hline
\end{tabular}

Dans cette étude, nous nous proposons de préciser les critères d'identification concernant les 3 genres Sphaerotheca, Leveillula et Erysiphe. Nous apporterons ensuite quelques données relatives à la biologie des 2 principaux oïdiums. Enfin, par un travail exclusivement basé sur $S$. fuliginea, l'oïdium le plus répandu en serre, nous tenterons de définir quelques facteurs favorables à la maladie.

Des essais de contaminations croisées, d'une part sur Cucurbitacées, d'autre part sur diverses plantes, préciseront les limites d'adaptation parasitaire des oïdiums étudiés.

\section{MATÉRIEL ET MÉTHODES}

Nous avons travaillé avec 2 populations d'oïdiums : l'une $S$. fuliginea, provenant de la variété de melon "Védrantais », l'autre, E. cichoracearum, provenant de tabac «Xanthi ». Chaque population est entretenue sur son hôte d'origine, dans des compartiments de serre bien isolés, à $20-30{ }^{\circ} \mathrm{C}$.

Dans le contexte actuel, il ne nous est pas possible de préciser à quelle race physiologique appartient la souche de $S$. fuliginea multipliée régulièrement sur melon («Védrantais »), sur courgette («Diamant»), sur pastèque (fruit vert à graines rouges) et sur concombre («Marketer »). Confrontée à la gamme d'hôtes différentiels proposée par THOMAs (1978), la souche utilisée ne correspond aux critères d'aucune des 3 races décrites jusqu'à présent (tabl. 1).

\section{A. Récolte des spores}

Les plantes destinées à fournir l'inoculum sont secouées une fois par jour de façon à éliminer régulièrement les spores des extrémités de chaînes, c'est-àdire les plus vieilles dont la germination risque d'être défectueuse.

Le jour où l'on souhaite récolter les spores, l'agitation s'effectue, soit au-dessus d'une lame sèche, soit au-dessus d'une boîte de Petri renfermant un milieu nutritif gélosé classique (pour un contrôle de germination), soit encore au-dessus des plantes à contaminer.

La récolte des spores peut également s'effectuer dans des conditions plus rigoureuses par agitation des organes malades dans de l'eau renfermant 1 p. 1000 d'un détergent anionique, le triton X 100. On contrôle alors aisément leur densité par comptage.

En aucun cas, il ne faut recueillir l'inoculum par grattage avec un pinceau, car on risque alors de décrocher des spores trop jeunes et, par conséquent, incapables de germer (ZARACOVITIS, 1964).

\section{B. Essais de contamination artificielle}

Pour contaminer, soit des organes foliaires en survie sur l'eau, soit des plantes entières, la pulvérisation d'une suspension de spores dans l'eau avec 1 p. 1000 de triton X 100 (40000 spores $/ \mathrm{ml}$ ) est préférable au saupoudrage parce qu'elle permet une meilleure répartition de l'inoculum. 
Le matériel végétal est ensuite placé dans un endroit peu lumineux, en l'absence d'hygrométrie saturante, dans une gamme de température comprise entre 20 et $30{ }^{\circ} \mathrm{C}$.

\section{Notations des symptômes}

Chaque organe en survie est noté séparément. Quand il s'agit d'une plante entière contaminée, on apprécie visuellement l'ensemble de la partie aérienne attaquée. Les lectures reposent sur l'attribution de notes de 0 à 5 en fonction de la surface envahie par le parasite :

0 : pas de symptômes ;

1 : de 0 à 20 p. 100 de la surface envahie ;

2 : de 20 à 40 p. 100 de la surface envahie ;

3 : de 40 à 60 p. 100 de la surface envahie;

4 : de 60 à 80 p. 100 de la surface envahie ;

5 : de 80 à 100 p. 100 de la surface envahie.

Les notes cumulées sont alors exprimées en p. 100 de sensibilité, 100 p. 100 correspondant à une plante envahie dans sa quasi totalité par l'oïdium.

\section{Conservation des souches}

En l'état actuel des connaissances, les oïdiums ne se conservent que sur du matériel végétal vivant, c'est-àdire sur feuilles en survie (valable uniquement pour S. fuliginea) ou sur plantes entières. Dans ce dernier cas, nous avons conçu un dispositif de cabines pressurisées décrit par ailleurs (FAUVEL \& MOLOT, 1985). Cet appareillage, simple et peu coûteux, mettant à l'abri de toute contamination extérieure, permet non seulement de produire du matériel sain, mais encore de conserver des souches d'oïdium aux caractéristiques bien établies.

\section{RÉSULTATS}

\section{A. Identification cryptogamique des différents oïdiums parasitant les cucurbitacées (tabl. 2)}

Etant donné l'impossibilité de reconnaître les différents oïdiums par leurs symptômes (sauf dans le cas de Leveillula où l'on observe la sortie des bouquets de conidiophores par les stomates), on recourt soit à l'examen des formes parfaites ou imparfaites du champignon, soit à l'utilisation de «plantes filtres».

\section{Observation des périthèces}

Sur Cucurbitacées, les formes parfaites sont très rares (HIRATA, 1968). Elles peuvent s'observer à l'automne, en fin de végétation, sur les organes malades. Ornementés de fulcres flexueux bruns, les périthèces sont de dimension très variable : les plus petits $(65-98 \mu \mathrm{m})$ appartiennent à $S$. fuliginea, les plus gros $(135-250 \mu \mathrm{m})$ à L. taurica, E. cichoracearum occupant une position intermédiaire. La dimension du périthèce traduit en général le nombre d'asques qu'il renferme : 1 chez $S$. fuliginea, 20 à 35 chez $L$. taurica. Le nombre d'ascospores par asque est lui-même très variable : en moyenne 2 (3) chez E. cichoracearum, 8 chez S. fuliginea, 2 chez L. taurica.

Le déterminisme de la formation des périthèces est inconnu et leur rôle dans la conservation du champignon très mal défini.

\section{Observation des formes asexuées}

Etant donné la rareté d'apparition des périthèces, plusieurs auteurs ont tenté de caractériser les oïdiums d'après leurs caractères conidiens (ZARACOVITIS, 1965 ; NARAYANASAMY \& RAMAKRISHAN, 1971; BOESEWINKEL, 1977, 1980 ; HAMMET, 1977 ; YARWOOD, 1978a ; VAN JAARSVELD, 1984).

TABLEAU 2

Quelques critères de différenciation des oüdiums sur Cucurbitacées. Some criteria for the identification of powdery mildew infections on cucurbits.

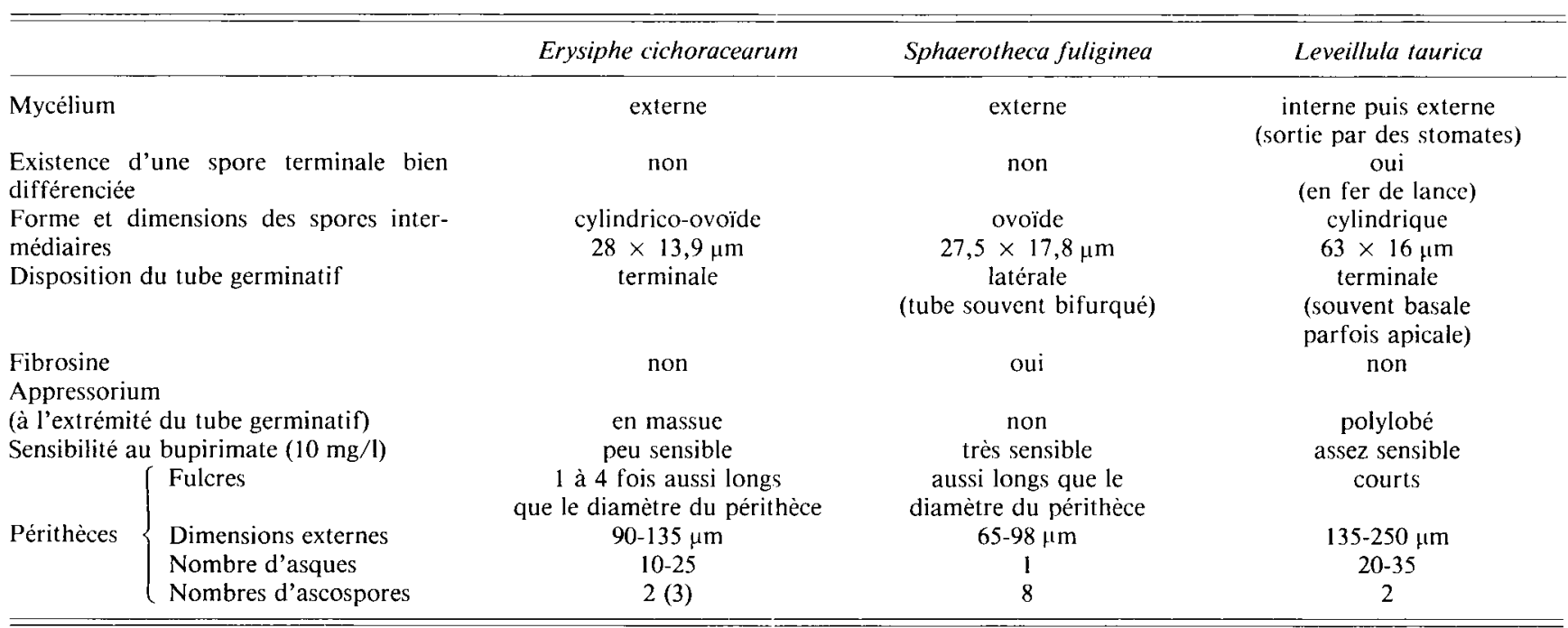


L'examen microscopique d'une population d'oïdiums conduit à distinguer 3 types de spores:

type ovoïde : genre Sphaerotheca;

type cylindrico-ovoïde : genre Erysiphe ;

type cylindrique : genre Leveillula.

En outre, à l'extrémité de la chaîne conidienne, L. taurica possède une spore terminale, nettement différente des autres, présentant un aspect pointu, en fer de lance.

Le genre Leveillula mis à part, il n'existe pas entre les oïdiums de différences marquées dans les dimensions des spores. Toutefois, le rapport $\frac{\text { longueur }}{\text { largeur }}$ permet de séparer $S$. fuliginea de E. cichoracearum : dans le $1^{\text {er }}$ cas, il est voisin de 1,6 ; dans le $2^{\text {e }}$ de 2,1 (NAGY, 1970 ; SOKOLOV, 1978 ; LEBEDA, 1983).

Par action de la potasse à froid à 3 p. 100, il apparaît, à l'intérieur des spores de $S$. fuliginea, des grains de fibrosine d'aspect très divers : corps sphériques, fines aiguilles, formations coniques, bâtonnets en croissant, etc... . De telles figures sont inexistantes dans les genres Erysiphe et Leveillula (HOMMA, 1937 ; BALLANTYNE, 1975 ; YARWOOD, 1978a).

Le mode de germination des spores apporte des éléments d'identification intéressants. Chez Erysiphe et Leveillula, le tube germinatif apparaît toujours en position terminale, c'est-à-dire du côté de l'apex, ou du côté de la base (ce dernier cas étant le plus fréquent chez Leveillula); chez Sphaerotheca, la spore germe latéralement, et souvent, le tube germinatif se ramifie en 2 branches (CRÜGER \& MEYER, 1976; SCHLÖSSER, 1976).

En fonction de la plante qui fournit les spores, une grande diversité s'observe dans la morphologie des tubes germinatifs ; 23 types différents ont été décrits chez S. fuliginea (IALONGO, 1981).

A l'extrémité du tube germinatif, il peut apparaître en quelques heures un appressorium en massue (E. cichoracearum) ou de forme polylobée ( $L$. taurica). En revanche, cette formation ne semble pas exister chez S. fuliginea (BOESEWINKEL, 1977).

Enfin, il existe entre les différents oïdiums, une sensibilité variable aux fongicides (CRÜGER \& MEYER, 1976). Nous avons montré que les Erysiphe sont plus résistants au bupirimate à $10 \mathrm{mg} / \mathrm{l}$ que Sphaerotheca et Leveillula.

\section{Utilisation de "plantes filtres"}

Indépendamment des critères que nous venons d'indiquer, il est possible, à l'aide de plantes-hôtes différentielles, de reconnaître $E$. cichoracearum de S. fuliginea.

Le $1^{\text {er }}$ oïdium est hébergé par Coccinia cordifolia (KHAN, 1978) ou par Aster dumosus L. (NAGY, 1972) alors que le $2^{\mathrm{e}}$ est le seul à infecter Lagenaria leucantha Rusby (KHAN, 1978).

\section{B. Quelques différences dans la biologie de Sphaero- theca fuliginea et d'Erysiphe cichoracearum}

\section{Données bibliographiques}

Les oïdiums des Cucurbitacées sont en général assez peu exigeants vis-à-vis de la température. Sur concom- bre, on a signalé un meilleur développement de $S$. $f u$ liginea à des températures inférieures à $21^{\circ} \mathrm{C}$, la gamme $15-21{ }^{\circ} \mathrm{C}$ paraissant plus favorable à la maladie que celle de $21-27^{\circ} \mathrm{C}$ (MUNGER, 1979). Les spores d'E. cichoracearum germent et se développent dans une gamme de températures plus large que celles de S. fuliginea (NAGY, 1976).

Vis-à-vis de l'hygrométrie, $E$. cichoracearum réclame des conditions assez sèches, tandis que $S$. fuliginea semble stimulé en présence d'une humidité élevée. Les spores d'E. cichoracearum peuvent germer à moins de 94 p. 100 d'humidité relative, celles de $S$. fuliginea exigent une hygrométrie saturante (NAGY, 1976 ; YARWOOD, 1978b).

En condition froide et humide, $S$. fuliginea sporule moins, mais conserve une agressivité élevée ; en revanche, par temps chaud et sec, les spores sont nombreuses, mais peu pathogènes (BASHI \& AUST, 1980).

Dans tous les cas, la réussite d'une contamination artificielle exige au départ une hygrométrie assez forte pendant $24 \mathrm{~h}$ suivie de conditions plus sèches dans les jours qui suivent (ABIKO \& KISHI, 1979).

\section{Observations personnelles}

Sur lames de verre sèches ou en boîtes de Petri renfermant un milieu nutritif gélosé, les spores d'E. cichoracearum germent toujours mieux que celles de $S$. fuliginea. En moins de $24 \mathrm{~h}$, les tubes germinatifs ont atteint leur taille définitive.

Les contaminations artificielles par E. cichoracearum et par S. fuliginea de leurs hôtes respectifs (tabac ou melon) présentent de nettes différences selon les conditions dans lesquelles on opère. Après pulvérisation de spores sur des feuilles en survie, E. cichoracearum se développe difficilement en une dizaine de jours sur tabac (il importe alors de laisser le couvercle de la boîte de Petri entrouvert), tandis que $S$. fuliginea donne des symptômes sur melon en $5 \mathrm{j}$; par contre, sur plantes entières, l'infection du tabac par $E$. cichoracearum est plus rapide à réaliser que celle du melon ou de la courgette par $S$. fuliginea.

Pour compléter cette étude, il conviendrait de réaliser la contamination du melon avec une souche d' $E$. cichoracearum adaptée à cette plante.

\section{Essais de contaminations croisées avec différents hôtes}

Les oïdiums sont en général réputés pour leur haute spécificité parasitaire; si $E$. cichoracearum et $S$. fuliginea peuvent se rencontrer sur un grand nombre de plantes, l'une ou l'autre espèce, récoltées sur un hôte donné, est souvent incapable de contaminer un hôte différent, appartenant parfois à la même famille botanique.

Des essais ont été réalisés sur feuilles en survie et sur plantes entières.

\section{Sur feuilles en survie}

Le transfert de l'oïdium du tabac ( $E$. cichoracearum) serait possible chez la courgette, le concombre et le melon (DECKENBACH, 1924, cité par COLE, 1978). Des travaux plus récents signalent le passage sur Cucumis anguria L. et Cucurbita maxima Duch. 
(MARCELLI, 1949) ainsi que sur pomme de terre (HoPKINS, 1948, cité par COLE, 1978).

$E$. cichoracearum, largement répandu sur Cucurbitacées en Hongrie, peut contaminer sous serre Aster dumosus L. et Aster novi-belgi L. (NAGY, 1972).

Nous mêmes, à partir de 5 souches différentes d'Erysiphe (tabac, courgette, Aster sp., Lactuca serriola $=L$. scariola $\mathrm{L}$. et Senecio vulgaris L.), avons réalisé des contaminations de feuilles en survie de diverses Cucurbitacées, la souche courgette paraissant la plus polyphage (tabl. 3).

Par ailleurs, disposant d'un inoculum abondant de S. fuliginea provenant de melon, nous avons pu transmettre la maladie à des feuilles en survie de mâche (Valerianella olitoria Poll.), de souci (Calendula officinalis L.), de séneçon ( $S$. vulgaris) et de diverses salades (scarole, frisée, mais pas la laitue). Le retour sur cucurbitacées est également possible.

Signalons enfin que $E$. polygoni récolté sur la renouée des oiseaux (Polygonum aviculare L.) n'est pas capable, dans nos conditions expérimentales, de contaminer les Cucurbitacées.

\section{Sur plantes entières (tabl. 4)}

Une population de $S$. fuliginea provenant de melon est multipliée respectivement sur melon, courgette, pastèque et concombre. L'inoculum issu de chacun de ces hôtes est, après ajustage à la même concentration de spores, pulvérisé sur des plantes au stade 5 feuilles de melon, courgette, pastèque et concombre.

Les 4 plantes-hôtes présentent des variations importantes dans la sensibilité à $S$. fuliginea : le melon et la pastèque montrent des symptômes assez atténués tandis que le concombre et surtout la courgette se révèlent très réceptifs vis-à-vis de l'oïdium.

Par ailleurs, il apparaît que le matériel cryptogamique récolté sur pastèque est le plus agressif, tandis que celui issu de concombre attaque peu les 4 cucurbitacées testées. Le pouvoir pathogène des spores provenant de melon et courgette se situe en position intermédiaire.

\section{TABLEAU 3}

Contamination de feuilles en survie par 5 souches $d$ 'Erysiphe cichoracearum.

Infection of detached leaves by 5 isolates of Erysiphe cichoracearum.

\begin{tabular}{|c|c|c|c|c|c|}
\hline & \multicolumn{5}{|c|}{ Erysiphe cichoracearum provenant de } \\
\hline & $\begin{array}{l}\text { Tabac } \\
\text { xanthi }\end{array}$ & $\begin{array}{l}\text { Cour- } \\
\text { gette }\end{array}$ & $\begin{array}{l}\text { Aster } \\
\text { sp. }\end{array}$ & $\begin{array}{l}\text { Lactuca } \\
\text { serriola }\end{array}$ & $\begin{array}{l}\text { Senecio } \\
\text { vulgaris }\end{array}$ \\
\hline $\begin{array}{c}\text { Cucumis melo } \\
\text { (melon) }\end{array}$ & & + & & & \\
\hline $\begin{array}{r}\text { Cucurbita pepo } \\
\text { (courgette) }\end{array}$ & + & + & + & & + \\
\hline $\begin{array}{l}\text { Citrullus vulgaris } \\
\text { (pastèque) }\end{array}$ & & + & & & \\
\hline $\begin{array}{l}\text { Cucumis sativus } \\
\text { (concombre) }\end{array}$ & & + & + & + & \\
\hline Coccinia cordifolia & + & + & & & \\
\hline
\end{tabular}

+ présence de symptômes.

\section{Influence de quelques facteurs sur les contamina- tions artificielles par $S$. fuliginea}

Indépendamment de la température et de l'hygrométrie, d'autres facteurs interviennent dans le développement de l'oïdium, en particulier l'alimentation de la plante et une infection par un virus.

\section{Alimentation de la plante (tabl. 5)}

Trois jours avant d'être contaminés, des plants de courgette et de tabac au stade 3-4 feuilles sont arrosés avec $50 \mathrm{ml}$ de solution nutritive $(*)$, cette opération étant répétée 2 fois par semaine pendant 3 semaines. Sept jours après la contamination, il n'y a pas encore de symptôme d'oïdium sur les courgettes témoins, tandis que celles alimentées avec de la solution nutritive sont déjà atteintes à 21 p. 100 ; sur tabac, l'infection est plus importante que sur courgette, mais on observe la même tendance. Après 3 semaines, les plantes convenablement alimentées manifestent une sensibilité à l'oïdium significativement supérieure à celle des témoins eau.

\section{TABLEAU 4}

Sensibilité à Sphaerotheca fuliginea de différentes cucurbitacées en fonction de l'origine de l'inoculum (contamination de plantes entières sous serre) en \% de surface foliaire colonisée.

Susceptibility to Sphaerotheca fuliginea of different cucurbits according to the inoculum source (inoculation of whole plants under greenhouse conditions), in \% of colonized leaf surface.

\begin{tabular}{lccccc}
\hline \hline & \multicolumn{5}{c}{ Sphaerotheca fuliginea provenant de } \\
\cline { 2 - 6 } Plante-hôte & Melon & $\begin{array}{c}\text { Cour- } \\
\text { gette }\end{array}$ & $\begin{array}{c}\text { Pastè- } \\
\text { que }\end{array}$ & $\begin{array}{c}\text { Con- } \\
\text { combre }\end{array}$ & $\begin{array}{c}\text { Moyen- } \\
\text { ne }\end{array}$ \\
\hline Melon & 6 & 9 & 11 & 4 & 7,5 \\
Courgette & 28 & 32 & 34 & 16 & 25,5 \\
Pastèque & 8 & 6 & 10 & 5 & 7,25 \\
Concombre & 15 & 22 & 29 & 12 & 19,5 \\
\hline Moyennes & 14,25 & 17,25 & 21,0 & 9,25 & \\
\hline
\end{tabular}

According to leaf area colonized, ratings from 0 to 5 were given : $0:$ no symptoms

$1: 0$ to $20 \%$ leaf area colonized

$2: 20$ to $40 \%$ leaf area colonized

$3: 40$ to $60 \%$ leaf area colonized

$4: 60$ to $80 \%$ leaf area colonized

$5: 80$ to $100 \%$ leaf area colonized

Data are cumulated and expressed as $\%$ of susceptibility, $100 \%$ representing a plant completely covered by powdery mildew.

(*) Engrais foliaire complet MAIRoL.

14 p. 100 d'azote ( 7 p. 100 sous forme nitrique et 7 p. 100 sous forme ammoniacale) ;

12 p. 100 d'acide phosphorique (100 p. 100 soluble dans l'eau) ;

14 p. 100 de potasse (sous forme de nitrate et sulfate; pas de chlore).

1 p. 100 de magnésie (sous forme de sulfate).

Oligoéléments présents :

Sulfate de manganèse, acide borique, sulfate de zinc, sulfate de cuivre, nitrate de cobalt, hormones de croissance, matières stimulantes (aneurine, lactoflavine, amide de l'acide nicotinique).

Au moment de l'emploi, on mélange $750 \mathrm{~g}$ de cette préparation à 51 d'eau. 
TABLEAU 5

Influence de l'alimentation de la plante (labac ou courgette) sur la sensibilité à l'ordiam (E. cichoracearum ou S. fuliginca), en \%. Effect of plant nutrition on susceptibility to powdery mildew (tobacco/E. cichoracearum, courgette/S. fuliginea), in $\%$.

\begin{tabular}{lcccccccc}
\hline & \multicolumn{3}{c}{ Solution nutritive } & \multicolumn{3}{c}{ Témoin eau } \\
\cline { 2 - 8 } & J 7 & J 14 & J 21 & J 7 & J 14 & J 21 \\
\hline $\begin{array}{l}\text { Tabac }(N . \text { tabacum }) \\
(\text { E. cichoracearum })\end{array}$ & 44 & 72 & 97 & 18 & 23 & 29 \\
$\begin{array}{l}\text { Courgette }(C . \text { pepo) } \\
(S . \text { fuliginea })\end{array}$ & 21 & 49 & 81 & 0 & 5 & 9 \\
\hline
\end{tabular}

$\mathrm{J} 7, \mathrm{~J} \mathrm{14,} \mathrm{J} 21$ : Lecture $7,14,21 \mathrm{j}$ après la contamination.

Measurements made $7,14,21$ days after contamination.

\section{Préinoculation virale}

Une rapide revue bibliographique montrant l'importance d'une préinoculation virale sur la sensibilité des plantes à l'oüdium (BLUMER et al., 1956; GOHEEN \& SCHNATHORST, 1961 ; KING et al., 1964 ; RUSSEL, 1969), nous avons tenté de mettre en évidence ce phénomène chez le melon "Védrantais ".

Des plantules au stade 2 cotylédons -1 feuille sont inoculées mécaniquement au niveau d'un cotylédon par l'un des 4 virus suivants : virus de la mosaïque de la pastèque 1 et 2 (WMV 1 et WMV 2), virus de la mosaïque du concombre (CMV) ou virus de la mosaïque jaune de la courgette (ZYMV).

Ces contaminations virales sont réalisées soit juste avant la pulvérisation de $S$. fuliginea (tabl. 6), soit 2 ou $6 \mathrm{j}$ avant (tabl. 7).

On constate qu'une préinoculation par les 4 virus (ce phénomène est particulièrement net dans le cas du CMV) entraîne une diminution de sensibilité des plantes à l'oïdium. Cet effet est encore plus accentué quand les virus sont apportés 2 ou mieux $6 \mathrm{j}$ avant la contamination par $S$. fuliginea.

Ces essais devront être confirmés par des observations au champ.

\section{DISCUSSION - CONCLUSION}

Dans les conditions culturales de la France, 2 champignons sont essentiellement responsables des attaques d'oödium sur Cucurbitacées : $S$. fuliginea et, à un degré moindre, E. cichoracearum.

Même en l'absence de périthèces, il est possible de distinguer les 2 espèces d'après l'aspect des oïdies, leur mode de germination et la présence de grains de fibrosine. Cette distinction apparaît d'autant plus fondée que la biologie des 2 champignons diffère, principalement en ce qui concerne les conditions d'hygrométrie : un climat sec est plus favorable au développement et à la sporulation de E. cichoracearum; en revanche, $S$. fuliginea, dont les conidies ne germent qu'en atmosphère saturée, semble mieux adapté aux conditions de serre.

Bien que les oïdiums soient classiquement reconnus pour leur grande spécificité d'hôte, nous avons mon-
TABLEAU 6

Sensibilité à Sphaerotheca fuliginea de la variété de melon « Védrantais" ayant subi une inoculation virale (sans attente entre les 2 contaminations) en $\%$.

Susceptibility of muskmelon « Védrantais » to S. fuliginea when coinoculated or not with a virus (in \%).

\begin{tabular}{lcc}
\hline & J 7 & J 14 \\
\hline Témoin non virosé & 22 & 64 \\
\hline WMV 1 & 4 & 56 \\
\hline WMV 2 & 3 & 48 \\
\hline CMV & 0 & 17 \\
\hline ZYMV & 5 & 45 \\
\hline \hline
\end{tabular}

J 7, J 14 : Lecture 7, $14 \mathrm{j}$ après la contamination par $S$. fuliginea. Measurements made 7, 14 days after contamination.

\section{TABLEAU 7}

Sensibilité à Sphaerotheca fuliginea de la variété de melon « Védran tais " atant subl the priinocutation virale $2(J-2)$ ou $6(J-6)$ jours aramt la contamination par l'oildium (J) en \%.

Susceptibiln! of mitustmelon " ledirantais " to Sphaerotheca fuliginea, after inoculation virus 2 or 6 days before the inoculation with the powdery mildew, in \%.

\begin{tabular}{lll}
\hline \hline Témoin non virosé & $\mathrm{J}$ & 56 \\
\hline \multirow{2}{*}{ WMV1 } & $\mathrm{J}-6$ & 38 \\
& $\mathrm{~J}-2$ & 47 \\
WMV2 & $\mathrm{J}-6$ & 38 \\
& $\mathrm{~J}-2$ & 43 \\
\hline \multirow{2}{*}{ CMV } & $\mathrm{J}-6$ & 16 \\
& $\mathrm{~J}-2$ & 22 \\
\multirow{2}{*}{ ZYMV } & $\mathrm{J}-6$ & 28 \\
& $\mathrm{~J}-2$ & 39 \\
\hline \hline
\end{tabular}

Lecture 14 jours après la contamination par $S$. fuliginea.

Measurements made 14 days after inoculation.

tré que, dans les conditions particulières d'organes en survie sur l'eau, il y avait possibilité de contaminations croisées. $S$. fuliginea provenant du melon peut passer sur la mâche, le souci, le séneçon et diverses salades ; par ailleurs, 4 souches différentes d'E. cichoracearum non originaires de cucurbitacées ont été capables d'infecter la courgette et le concombre. Ces résultats devront être confirmés par des contaminations de plantes entières, car cette question pose le problème des hôtes susceptibles de servir de sources d'inoculum (STONE, 1962).

Parmi les facteurs jouant un rôle important dans la réceptivité des Cucurbitacées aux oïdiums, nous avons étudié l'influence de l'alimentation de la plante et d'une préinoculation virale.

Les plantes ayant reçu de la solution nutritive sont nettement plus sensibles à l'oïdium. On savait déjà que les feuilles carencées en azote sont en général peu atteintes d'oïdium. Avec le couple Sphaerotheca morsuvae (Schw.) Berk. \& Curt. - groseillier, TEMMEN et 
al. (1980) ont montré que la fructification conidienne est abondante si les cellules sont turgescentes. Les oïdiums tirant leur nourriture de l'hôte par les haustoria, cette hypothèse demeure tout à fait vraisemblable.

Sur Vigna mungo, une faible fumure apportant moins de $30 \mathrm{~kg}$ d'azote à l'ha entraîne une réduction importante d'E. polygoni (NAWAZ \& NARAYANASAMY, 1983).

Une préinoculation virale, déclenchant des perturbations métaboliques importantes, joue également un rôle non négligeable dans le développement des oïdiums. Infecté localement avec le Tobacco Necrosis Virus (TNV) sur les cotylédons ou les feuilles inférieures, Cucumis sativus L. est protégé partiellement sur les feuilles supérieures vis-à-vis de $S$. fuliginea (BASHAN \& COHEN, 1983). Chez le melon, une contamination par le CMV réalisée juste avant la contamination par S. fuliginea ou au contraire $18 \mathrm{j}$ avant, entraîne dans le $1^{\text {er }}$ cas une réduction d'oïdium d'environ 30 p. 100 , dans le $2^{\text {e }}$ une réduction supérieure à
50 p. 100 ; l'infection virale limite la taille et le nombre des colonies d'oïdium (SHARMA, 1983).

En revanche, sur concombre, une attaque des racines par Phomopsis sclerotioides v. Kesteren déclenche une apparition plus précoce et des dégâts plus sévères de $S$. fuliginea (EBBEN \& SEARLE, 1976).

Le problème le plus important demeure sans aucun doute celui de la conservation du parasite. A notre connaissance, toutes les tentatives pour cultiver les oïdiums sur milieux gélosés nutritifs se sont soldées par un échec. Il faut toujours recourir à un support végétal vivant et, bien que nous ayons résolu un certain nombre de difficultés par la mise au point de cabines pressurisées (FAUVEL \& MOLOT, 1985) et le maintien de $S$. fuliginea sur feuilles en survie, cette obligation demeure assez contraignante. Des travaux doivent être poursuivis dans cette direction.

Reçu le 29 juillet 1985 Accepté le 2 décembre 1985

\section{RÉFÉRENCES BIBLIOGRAPHIQUES}

Abiko K., Kishi K., 1979. Influence of temperature and humidity on the outbreak of cucumber powdery mildew. Bull. Veg. ornamental. Crops Res. Stn. Jap., Ser. A 5, 167-176.

Angelov D., 1979. Study of the susceptibility of cucumber cultivars to the pathogen of powdery mildew (Sphaerotheca fuliginea Pol.). Gradina. Lozar. Nauka, 16 (2), 98-103.

Angelov D., Pethova T., 1979. Age resistance of cucumber to the pathogen of powdery mildew (Sphaerotheca fuliginea). Gradina. Lozar. Nauka, 16 (2), 93-97.

Ballantyne B., 1975. Powdery mildew on Cucurbitaceae : identity, distribution, host range and sources of resistance. Proc. linn. Soc. N.S.W., 99, 100-120.

Bashan B., Cohen Y., 1983. Tobacco necrosis virus induces systemic resistance in cucumbers against Sphaerotheca fuliginea. Physiol. Plant Pathol., 23 (1) 137-144.

Bashi E., Aust H. J., 1980. Quality of spores produced in cucumber powdery mildews (Erysiphaceae) Bot. Rev., 46, 167-224.

Pflanzenschutz., 87, 594-599.

Blumer S., Stalder L., Harder A., 1956. Über die gegenseitigen Beziehungen zwischen Gurkenmosaik und Gurkenmehltau. Phytopathol. Z., 25, 39-54.

Boesewinkel H. J., 1977. Identification of Erysiphaceae by conidia! characteristics. Rev. Mycol., 41, 493-507.

Boesewinkel H. J., 1980. The morphology of the imperfect states of powdery mildews (Erysiphaceae) Bot. Rev., 46, 167-224.

Cole J. S., 1978. Powdery mildew of lobacco, 447-472. In Spencer D. M. : "The powdery mildews », Acad. Press, London, 565 p.

Collingwood E. F., 1977. L. taurica sur concombre. Sénégal. F.A.O. Bull. phytosanit., 25 (3), p. 130.

Crüger G., Meyer E., 1976. Möglichkeiten zur Differenzierung der Erreger des Echten Mehltaus an Kürbisgewächsen, (Sphaerotheca fuliginea und Erysiphe cichoracearum). Nachrichtenbl. dtsch. Pflanzenschutzdienstes, Stuttgart, 28, 49-55.

Docea E., Fratila E., 1980. A new pathogen causing powdery mildew of cucumber in Romania. Lucrari stiintifice, B., 22, 165-168.

Ebben M. H., Searle L. M., 1976. Observations on the effect of black root rot (Phomopsis sclerotioides) on the susceptibility of cucumber to powdery mildew (Sphaerotheca fuliginea). Ann. appl. Biol., 83 (1), 169-172.

El Ammari S. S., Khan M. W., 1983. Leveillula taurica, powdery mildew on greenhouse cucumbers (Cucumis sativus) in Libya. Plant Dis., 67 (5), 553-555.

Fauvel C., Molot P. M., 1985. Une enceinte de conception fort simple, pour l'étude des oïdiums. Cahier techn. I.N.R.A., 11, 9-20.
Goheen A. C., Schnathorst W. C., 1961. Resistance to powdery mildew in leafroll-affected grapevines. Plant Dis. Rep., 45, 641-643. Golovin P. N., 1956. Monographic survey of the genus Leveillula Arnalud (parasitic fungi - fam. Erysiphaceae). Rev. appl. Mycol., 37, p. 400 (abstr.).

Gordeeva N. G., 1961. The relationship of ecological groups of melon to fungal diseases. Rev. appl. Mycol., 42, p. 356 (abstr.).

Hammarlund C., 1945. Beiträge zur Revision einiger imperfekten Mehltau-Arten Erysiphe polyphaga nov. sp. Bot. Not., 1, 101-108. Hammet K. R. W., 1977. Taxonomy of Erysiphaceae in New /ciiland. N. Z. J. Bot., 15, 687-711.

Hirata K., 1968. Notes on host range and geographic distribution of the powdery mildew fungi. Trans. mycol. Soc. Jap., 9, 73-88.

Homma Y., 1937. Erysiphaceae of Japan. J. Fac. Agric. Hokkatido Univ., 38, 183-461.

Ialongo M. T., 1981. Sulla forma del tubo germinativo conidico della Sphaerotheca fuliginea. Ann. Ist. sper. Patol. veget., VII, 87102 .

Janke Ch., Peter K., Hellwig A., 1977. Uber das Auftreten der Mehltaugattungen Erysiphe und Sphaerotheca an Gurken in der DDR. Arch. Phytopathol. Pflanzensch., Berlin, 13, 263-269.

Khan M. W., 1978. Coccinia cordifolia and Lagenaria leucantha, differential hosts for powdery mildews of cucurbits. Plant Dis. Rep., 62 (2), 161-164.

Khan M. W., 1983. The identity of powdery mildew of cucurbits ; a critical appraisal. Acta Bot. Indica, 11, 97-126.

King L. N., Hampton R. E., Diachun S., 1964. Resistance to Erysiphe polygoni of red clover infected with bean yellow mosaic virus. Science, 146, 1054-1055.

Lebeda A., 1983. The genera and species spectrum of cucumber powdery mildew in Czechoslovakia. Phytopathol. Z., 108, 71-79.

Marcelli E., 1949. Preliminary observations on specialization in tobacco powdery mildew. Notizario. Mal. Piante, 6, 19-21.

Munger H. M., 1979. The influence of temperature on powdery mildew resistance in cucumber. Plant Breed. Abstr., 50, 109-119.

Nagy G. S., 1970. Die Identifizierung des Mehltaus der Kürbisgewächse auf Grund der Konidienmerkmale. Acta phytopathol. Acad. Sci. hung., 5, 231-248.

Nagy G. S., 1972. Studies on powdery mildews of cucurbits. I. Host range and mainicnance of Sphuerotheca fuliginea and Lrysiphe $p$. under laboratory and glasshouse conditions. Acta phytopathol. Acad. Sci. hung., 7, 415-420.

Nagy G. S., 1975. Revision of the powdery mildew species Erysiphe 
polyphaga Hamm. Acta phytopathol. Acad. Sci. hung., 10, 377388.

Nagy G. S., 1976. Studies on powdery mildews of cucurbits. II. Life cycle and epidemiology of Erysiphe cichoracearum and Sphaerotheca fuliginea. Acta phytopathol. Acad. Sci. hung., 11, 205-210.

Narayanasamy P., Ramakrishnan K., 1971. Powdery mildews of Coimbatore, Madras State. J. Madras Univ., B., 37, 84-99. Abstr. in Rev. Plant Pathol. (1974), 53, 839.

Nawaz R. M. S., Narayanasamy P., 1983. Influence of host nutrition on powdery mildew disease development in black gram. Madras Agric. J., 70 (1), 57-58.

Pyzhenkov V. I., 1977. Initial material for breeding cucumber in the non-chernozem zone of the Russian S.S.R. Tr. prikl. Bot. Genet. Sel., 60 (3), 119-132.

Russel G. E., 1969. Specific interactions between virus and fungus diseases in sugar beet. J. int. Inst. Sugar Beet Res., 3, 175-181. Abstr. in Rev. Plant Pathol. (1970), 49, 596.

Schlösser E., 1976. Vorkommen und Verbreitung der beiden Arten des Gurkenmehltaus, Sphaerotheca fuliginea und Erysiphe cichoracearum, in der Bundesrepublik Deutschland. Meded. Fac. Landbouwet Rijksuniv., Gent., 41, 539-544.

schroeter J.. 1893. Pilse. In 1: Cohn : "Kryprogamen-Flora von Schlesien ». Breslau, J. U. Kern, 1908, vol. 3 (2) 2, 229-249.

Sharma O. P., 1983. Interaction of cucumber mosaic virus and Sphaerotheca fuliginea in muskmelon. Sci. Cult., Punjab Agric. Univ., India, 49 (5), 139.

Sitterly W. R., 1978. Powdery mildews of cucurbits, 359-379. In Spencer D. M. : «The powdery mildews ». Acad. Press. London, $565 \mathrm{p}$.

Sokolov Y. V., 1978. Identification of powdery mildew causal agents on Cucurbitaceae in Astrakhan region. Mikol. Fitopatol., 12, 264-266.
Stone O. M., 1962. Alternate hosts of cucumber powdery mildew. Ann. appl. Biol., 50, 203-210.

Tarr S. A. J., 1955. Diseases of economic crops in the Sudan. F.A.O. Plant Prot. Bull., 3 (8), 113-116. Abstr. in Rev. appl. Mycol., 35, 880 .

Temmen K. H., Gruppe W., Schlosser E., 1980. Investigations on the resistance of plants to powdery mildew. III. Basis for the horizontal resistance of Ribes cv. to Sphaerotheca mors-uvae. Z. Pflanzenkr. Pflanzenschutz, 87 (3), 129-136.

Thomas C. E., 1978. A new biological race of powdery mildew of cantaloups. Plant Dis. Rep., 62 (3), p. 223.

Tramier R., 1963. Etude préliminaire du Leveillula taurica (Lev.) Arn. dans le midi de la France. Ann. Epiphyt., 14 (4), 355-369.

Van Jaarsveld A. B., 1984. Powdery mildew fungi in South Africa. Phytophylactica, 16, 155-166.

Viennot-Bourgin G., 1949. Les champignons parasites des plantes cultivées. Ed. Masson, Paris, $1850 \mathrm{p}$.

Viennot-Bourgin G., 1956. Mildious, ö̈diums, caries, charbons et rouilles des plantes de France. Encyclop. mycol. XXVII, 317 p., 89 pl. P. Lechevalier.

Viennot-Bourgin G., 1971. Erysiphales nouvelles ou peu connues en France. Ann. Phytopathol., 3, 337-352.

Yarwood C. E., 1978a. History and taxonomy of powdery mildew, 1-38. In D. M. Spencer : "The powdery mildews ». Acad. Press, London, $565 \mathrm{p}$.

Yarwood C. E., 1978b. Water stimulates Sphaerotheca. Mycologia, 70 (5), 1035-1039.

Zaracovitis C., 1964. Factors in testing fungicides against powdery mildews. The germination of the conidia in vitro. Ann. Inst. Phytopathol., Benaki, NS 6, 73-106.

Zaracovitis C., 1965. Attempts to identify powdery mildew fungi by conidial characters. Trans. Br. Mycol. Soc., 48, 553-558. 